\title{
Clinical academic careers for general practice nurses: a qualitative exploration of associated barriers and enablers
}

\section{Sarahjane Jones $\mathbb{}$}

Associate Professor, School of Health, Science and Wellbeing, Staffordshire University, UK

\section{Andrew Bradbury}

Health Tutor, Health and Education Department, CU Scarborough, Birmingham City University, UK

\section{Sue Shortland}

Associate Professor, School of Nursing and Midwifery, Birmingham City University, UK

\section{Fraser Hewett}

GP Partner, Manor Practice, Sutton Coldfield, UK

\section{Karen Storey}

Primary Care Nursing Lead, NHS North of England, UK

\begin{abstract}
Background: The delivery of research in healthcare in the UK is dependent on a subgroup of clinicians - clinical academics - who concurrently engage in clinical practice and academic activities. The need to increase access to such roles for general practice nurses has been identified, although the need for a robust career framework remains.

Aims: This study, with a qualitative interview and focus group design, aimed to explore the concept of clinical academic careers for general practice nurses by identifying barriers and enablers associated with pursuing and performing such roles.

Methods: General practice nurses $(n=18)$ and general practitioners $(n=5)$ engaged in either an audio -recorded interview or focus group. Verbatim transcripts were subjected to thematic analysis.

Results: Four themes were identified: awareness and understanding; career pathway; personal and professional attributes; and organisational factors. Awareness and understanding were
\end{abstract}


generally poor. Participants suggested that the career pathway was unclear, although it was generally assumed that such roles were 'out of reach' and require a minimum of Master's level education. An interest in research and the confidence to perform such duties were reported as the required personal and professional attributes. Organisational factors included the need for employers to understand the value and benefit of general practice nurse clinical academic roles, along with ensuring that the inevitable competing demands of such a role were appropriately managed.

Conclusions: This study highlights the difficulties faced by general practice nurses wishing to pursue a clinical academic career. Academia is seemingly placed on a pedestal, emphasising the need to embed research training early in nursing education to alter general practice nurse perceptions that clinical academic roles are unobtainable. The development of a robust career pathway for general practice nurse clinical academic roles may have a positive impact on the retention of experienced general practice nurses and attract newly qualified nurses. This research provides evidence as to the need for one.

\section{Keywords}

clinical academic careers, general practice nurse, research nurse

\section{Background}

There is increasing demand on healthcare globally and the National Health Service (NHS) in England is no exception. Over the past 5 years, attendances at major accident and emergency (A\&E) departments have risen by $10.3 \%$, and the number of people on the waiting list for consultant-led treatment has risen by $42 \%$ (Baker, 2019). Consequently, 4-hour waits in $\mathrm{A} \& \mathrm{E}$ are at an all-time high, as is the waiting list for consultant-led treatment and the time to treatment, and this was before the global coronavirus pandemic. This comes during a time when there have been increases in the absolute numbers across most clinical professions, but this increase is not in keeping with the growth in demand. The issue is further compounded when the volume of posts generated is greater than the supply of clinical professionals to fill them (Health Education England, 2017a). Between July and September 2018, there were nearly 94,000 full-time equivalent advertised vacancies in hospital and community services in England, equating to an $8 \%$ shortfall, with nursing and midwifery having nearly 40,000 vacancies (Rolewicz and Palmer, 2019). Challenges in workforce retention add to the vacancy problem, with a growing number of nurses leaving the profession early for reasons other than retirement (Health Education England, 2017a). These challenges exist in the context of growing expectation for accessible, high quality and safe care.

In an increasingly resource-constrained environment relative to demand, increasing research delivery is one mechanism that could alleviate challenges to improving care quality. The relationship between research delivery and patient outcomes in healthcare is well established. A systematic review and meta-analysis of international studies found that patients not only benefit directly from participation in research, achieving improved clinical outcomes (Nijjar et al., 2017), but also more generally at the organisation level. Patient populations achieved better outcomes in organisations with high research participation irrespective of their own participation in research studies (Downing et al., 2017). The 
delivery of research in healthcare in the UK is dependent on a subgroup of clinicians known as clinical academics who concurrently engage in clinical practice and academia (Baltruks and Callaghan, 2018).

Clinical academics are essential in both the development of real-world relevant, timely research and the translation of research back into practice, and form a pivotal component in the UK government's life science industrial strategy (Bell, 2017; HM Government, 2019). However, despite the recognition for there to be a clearer career pathway for nurses involved in clinical research for over a decade (UK Clinical Research Collaboration, 2006), and some progress having been made (Department of Health, 2012), the proportion of clinical academics in nursing, midwifery and allied health professions remains low at $0.1 \%$, (Dickinson et al., 2017) in comparison to $4.6 \%$ of the medical consultant workforce (Fisher et al., 2017).

The primary care workforce in England is no exception to the workforce challenges. A $1.7 \%$ decline in the number of full-time equivalent general practitioners (GPs) between September 2017 and 2018 (NHS Digital, 2018), coupled with a survey that reported that $39 \%$ of GP respondents planned to leave 'direct patient care' by 2022 (Gibson et al., 2017) makes for significant workforce challenges in general practice. A similar picture exists in general practice nursing across the UK; 33.4\% of general practice nurses (GPNs) are due to retire by 2020 (Bradby and McCallum, 2019) and over half of all GPNs in Scotland are over 50 years old (Innes, 2019).

GPNs are recognised as being key to the delivery of primary care services in the General Practice Forward View (NHS England, 2016). In an effort to address the challenges in the workforce, the General Practice Nursing Workforce Development Plan (Health Education England, 2017b) identified some key steps necessary to move forward including increased access to clinical academic careers. Subsequently, the NHS England 10-point action plan reiterated the need to develop clinical academic careers at point eight of the plan (NHS England, 2017). However, to realise opportunities for GPNs to take up clinical academic posts and develop a robust and fit-for-purpose career framework, evidence is needed to understand the barriers and enablers to a clinical academic career in the general practice setting and specifically for GPNs. A rapid evidence assessment undertaken to explore the available literature on GPNs and clinical academic nurses in the UK only found two articles that discussed GPNs (Bradbury et al., 2021).

Thus, this study aimed to explore the concept of clinical academic careers for GPNs through investigation of the barriers and enablers related to the development and functionality of the role, along with the associated potential benefits for general practice.

\section{Methods}

This paper details the qualitative element of a wider mixed -methods study within in a critical realist paradigm. Critical realism presents a philosophical paradigm that can accommodate mixed -methods studies that can be used to explore the underlying mechanisms that can generate events (Danermark et al., 2002). Bhaskar (2008) promotes the belief that in order to understand a phenomenon, you must understand these underlying mechanisms in addition to the traditional observable events sought in positivist paradigms. This paper presents the qualitative exploration of the potential underlying conditions for GPNs in pursing and performing clinical academic careers as a means to exploring the observable events; that clinical academic career uptake is lower in nursing than in medicine. GPs and GPNs were recruited from within the West Midlands of England through a combination of purposive 
and snowball sampling. Participants were recruited from across the experience spectrum, including both experienced clinicians and those new to the general practice setting. Participants provided informed consent to take part in an audio -recorded interview or focus group, with recordings transcribed and resultant transcripts subjected to a process of thematic analysis (Braun and Clarke, 2006). All participants were analysed as a single unit, with any professional differences documented. A semi-structured interview schedule was used and is available on request.

The research team comprised a multidisciplinary group of individuals who reflected the professions being investigated including GPNs and a GP, and two researchers without clinical registration. One team member provided the function of key stakeholder engagement to support in the development of the interview schedule and interpretation to improve trustworthiness and credibility. This study reports using the standards for reporting qualitative research (O’Brien et al., 2014).

\section{Results}

Three focus groups comprising a total of 12 registered nurses, and one-to-one interviews with six registered GPNs and five GPs were undertaken over a 6-week period in February and March 2019. The three focus groups comprised nurses new to the general practice role studying on the fundamentals of general practice nursing course (focus group A); GPNs who were studying for an advanced clinical practice award (focus group B); and GPNs working in general practice (focus group C). All of the nurses in the focus groups and interviews were currently employed as GPNs in the general practice setting. The breakdown of these focus groups and interviews and the related demographic data can be found in Table 1.

Following an iterative cycle of thematic analysis, four main themes were identified: awareness and understanding; career pathway; personal and professional attributes; and organisational factors.

\section{Awareness and understanding}

Awareness of the clinical academic role appeared to be relatively poor; most participants had never worked with anyone in a clinical academic post. However, participants had a general understanding of the term and were able to surmise the fundamental principles of the role. This lack of awareness of the role translated into difficulties in identifying opportunities, with all of the nurse participants never having considered the career as an option.

I wouldn't have even thought about it. So, it's raising awareness that I suppose it could be that it is an option, yeah. (Student GPN focus group)

The solitary nature of the GPN role was cited as one reason why GPNs might be less likely to learn of such roles, with limited opportunities to speak with colleagues in the working week and decreasing access to continuing professional development (CPD) and professional networks contributing further to the isolation. Exposure to how research relates to everyday clinical practice and role models in general practice were felt to be lacking. Participants thought that in hospital settings there would be more exposure to clinicians in clinical academic posts.

You know, so there are some clinical specialities, like oncology, for example, where loads of consultants will have PhDs, and so for them to think about doing a $\mathrm{PhD}$ isn't that big of a 
Table I. Demographic data.

\begin{tabular}{|c|c|c|c|}
\hline Current role & Age (years) & $\begin{array}{l}\text { Years as registered } \\
\text { nurse/doctor }\end{array}$ & $\begin{array}{l}\text { Time in general } \\
\text { practice }\end{array}$ \\
\hline \multicolumn{4}{|c|}{ Focus group $A, G P N$ students } \\
\hline Student GPN & 36 & 16 & $<12$ months \\
\hline Student GPN & 43 & 1 & $<12$ months \\
\hline Student GPN & 22 & 2 & $<12$ months \\
\hline Student GPN & 23 & 2 & $>12$ months \\
\hline Student GPN & 44 & 18 & $>12$ months \\
\hline Student GPN & 33 & 6 & $>12$ months \\
\hline \multicolumn{4}{|c|}{ Focus group $B$, GPNs studying for ACP award } \\
\hline Student ANP GPN & 36 & 14 & 2 years \\
\hline Student ANP GPN & 48 & 27 & 18 years \\
\hline Student ANP GPN & 35 & 13 & 2 years \\
\hline \multicolumn{4}{|l|}{ Focus group C, GPNs } \\
\hline FG GPN & - & 32 & 18 years \\
\hline FG GPN & 29 & 9 & I month \\
\hline FG GPN & 53 & 32 & 4 years \\
\hline \multicolumn{4}{|l|}{ Interviews } \\
\hline ACP GPN & 48 & 22 & - \\
\hline GP & 58 & 36 & 30 years \\
\hline GP & 54 & 31 & 27 years \\
\hline GP & 59 & 36 & 32 years \\
\hline GP & 35 & 12 & 6 years \\
\hline GP clinical academic & 37 & 13 & 18 months \\
\hline
\end{tabular}

ACP: advanced clinical practice; ANP: advanced nurse practitioner; GP: general practitioner; GPN: general practice nurse.

mental step. Whereas, if you're in a profession, like general practice, or primary care nursing, you don't have as many of your senior colleagues who have gone through that process. (GP5)

This lack of exposure was further compounded by the absence of nurse leaders in primary care promoting the clinical academic career pathway.

I think there should be more nurses high up within CCGs [clinical commissioning groups] that are definitely pushing the agenda and talking about it. (GPN student)

As the primary employers of GPNs, GPs' awareness and understanding of the GPN clinical academic role was considered essential in enabling GPNs to take up such roles. There was a large emphasis placed on the need for evidence as to the value of these roles, and that this message would need to be communicated explicitly and consistently to facilitate any discussion of the role. Limited awareness of the value and potential of GPNs pursuing clinical academic careers was evident in most of the interviews with GPs. However, two of the GP participants could envisage the value of a GP clinical academic, but were unsure of the significance/worth of GPN clinical academic roles.

I understand much more readily what the GP academic could offer and probably less likely to understand what a nurse academic would offer. I'm thinking in terms of as employer, the time that will be taken away. (GP2) 
The awareness of general negativity for the GPN clinical academic role was expressed by both GPN and GP participants. It was evident that there was a need for a change in attitudes and understanding throughout the general practice workforce so as to instil a positive environment and for opportunities to be realised.

\section{Personal and professional attributes}

There was a general consensus that the main driver for a GPN to consider a clinical academic role was a personal interest in research and the confidence one could develop the necessary skills even to consider applying for such a post. Participants expressed the need for a personal interest in research and the development of research skills to be nurtured from pre-registration and through post qualification education.

It was suggested, therefore, that confidence in one's ability to pursue research skills needed to be developed from the start of a nursing career to embed aspiration and expectations in new nurses that a clinical academic career was a viable, achievable and valid career goal.

I think maybe... when we're doing our initial nurse training to have researchers come in and discuss it. (GPN student)

However, many participants felt that the nature of the GPN role of autonomy and the personal characteristic of being self-motivated lent themselves well to the clinical academic role. Furthermore, the nature of the work of a GPN meant that they were in a good position to be able to identify patient-relevant research.

I think so because if we're working there we... know the flaws, we know when certain processes aren't working very well. So, it... you highlight that and you think, I need to change that. (GPN student)

However, while it was felt that a GPN would not necessarily need to be working at an advanced level of clinical practice, a suitable nurse for a clinical academic career would need to be experienced and confident in their clinical ability. There was concern that a focus on research would mean that a GPN would fall behind in terms of clinical progression.

I think one is making sure that you're giving yourself enough time to progress in your clinical career. Making sure that you're up to date with your CPD. (GP5)

There was, therefore, general agreement from the GPN participants that they had a lot to offer a clinical academic role, but lack of research knowledge and ability in their education and training impacted on their confidence to consider the role.

\section{Career pathway}

The lack of research ability and perception that clinical academic careers were out of reach of many GPNs was reflected by the absence of a formalised clinical academic career pathway for GPNs. Furthermore, to some extent, GPNs perceived that their career pathway was developed according to the needs of the practice, over and above their aspirations.

Because there's so much disparity in general practice about what your actual career path will be then you kind of either shape it yourself or the GP shapes it for you. (GPN student) 
There was a general consensus from GPN participants that a nurse would need to be educated to at least Master's level before considering a clinical academic career, suggesting that attainment of education level correlated with ability and disregarded an individual's potential early in their clinical career. Furthermore, the misconception of one GP, which possibly reflects wider opinion in medicine, that nurses are less academically competent than their medical colleagues by virtue that they are nurses rather than doctors would not be helpful in increasing uptake of nurses generally into clinical academic roles, and potentially be more damaging in a setting where the doctors with these opinions are simultaneously the employers of nurses.

I think by natural selection... that the more academic people are probably more steered towards medicine than nursing, I would suppose that's the case. (GP2)

There were limited opportunities for GPNs to develop capability in research over time. This, coupled with high volume workload and expectation that the development of clinical skills would be prioritised, impacted on participants' beliefs that clinical academic careers for GPNs were not a very viable option.

\section{Organisational factors}

Organisational factors featured heavily as barriers to clinical academic roles in general practice nursing. From the GP perspective, assurance on the value and benefits of such a role is required if they are to be expected to pay for it and indicated a preference for centrally funded roles.

As an employer I wouldn't want to be paying for another activity... from which I cannot derive direct benefit. So I see potential negatives and I see potential gains but I think the gains are less immediately obvious to me than from the GP academics. (GP2)

The division of time between clinical and academic aspects was of concern to both GPs and GPNs. There were fears from the GPNs that the academic element would be routinely compromised in order to meet increasing patient demand and staff shortages, while GPs felt that GPNs needed a minimum amount of clinical time to ensure maintenance of clinical skills and raised concerns around continuity of care for patients in the practice.

What about in the clinical areas, if the clinical load gets busy, you know, somebody's off sick or two people are on holiday, what happens to their academic bit? (ANP)

I think you have to have specific clinical days and specific research days, and your research days you have to be physically away, so that means usually at the university which you do your academic role at. Because, I think if your clinical colleagues see you around they will, inevitably, rope you into things while you're there and you will simply not have the time and head space to be doing any academic work. (GP5)

\section{Discussion}

The four themes presented all identify various barriers to the development of clinical academic careers for GPNs. In combination, they highlight the difficulties an individual would encounter in attempting to pursue this career pathway. Furthermore, the themes all demonstrate the culture around clinical research in nursing generally, and specifically in 
general practice. Inevitably the current high workload and dwindling workforce impacts on the enthusiasm of GPs and GPNs towards the development of clinical academic careers for GPNs but there is also an underlying negativity towards the feasibility of GPNs taking up such roles. This culture, in some places, is detrimental and even potentially toxic to the establishment and success of these roles in general practice nursing.

Academia was, to some extent, placed on a pedestal and this generated two positions regarding the suitability of GPNs for a clinical academic role. Nurses themselves tended to explain their reluctance or aversion to such roles in the context of confidence and modesty, that is, not having enough confidence or surplus modesty to put themselves forward for the 'superior' clinical academic role. This was further reinforced by the lack of research training in the early education of nurses, meaning that nurses did not feel adequately skilled to make the move into academia and consequently research can be something that nurses fear.

This position would not be helped by views potentially held by GPs that suggest that nursing as a profession is self-excluded from research because had they been academically competent, they would have been doctors. This is in contradiction to the current debate on the education of nurses. Evidence suggests that the registration status of the nursing profession, which requires an undergraduate level qualification, makes a significant contribution to patient safety in comparison to the non-registered nursing workforce (Aiken et al., 2017; Griffiths et al., 2018; Leary et al., 2016). Studies have also demonstrated that there is no difference between nurses and doctors in the competence of traditionally doctor-specific activities such as diagnosing (Pirret et al., 2015), running clinics (Larrson et al., 2014) and endoscopy (Centre for Reviews and Dissemination, 2011). However, it is important to recognise that nurses are not a cheaper alternative to doctors, but rather a valuable profession with an important role to play in delivering high quality, safe care to patients (Leary, 2012). However, there remain issues with the image of nursing perceived to be both a profession for women (MacWillians et al., 2013) and one that does not value the specialisms or advanced roles in nursing with protected titles, which potentially undermines confidence in the profession (Leary et al., 2017).

Further debate exists around the academic credibility of nursing. The credibility of the nursing professoriate in the UK has been questioned (Watson and Thompson, 2008; Watson et al., 2017); however, there appears to be consensus on the need for nursing leaders, such as those in chief nurse positions, to work 'hand in glove' to move forward the academic evidence base to 'address local service needs whilst being of an internationally excellent standard' (Cannaby et al., 2017). However, how nurses gain these academic skills while pursuing a clinical career remains to be determined as the career pathway for senior leadership roles does not currently require academic competence (Watson and Thompson, 2008); rather this is an aspiration and while only $0.1 \%$ of the non-medical workforce comprises clinical academics careers, an enormous investment is required to parallel the $4.6 \%$ of the medical workforce in clinical academic roles (Baltruks and Callaghan, 2018; Dickinson et al., 2017). It is, therefore, hardly surprising that this study highlighted a lack of role models in senior leaders in terms of research activity.

The national contract for consultant medics in England, in which academic pay is matched to the NHS pay structure (British Medical Association, 2020), is likely to go some way in supporting the larger proportion of medical academics. The importance of this offer is reinforced by the recent support of an equivalent offer by the Universities and Colleges Employers Association (British Medical Association, 2019) for medical clinical academics in training (those below consultant pay grade). In comparison, the absence of 
such an offer in nursing and other professions allied to health is indicative of the perceived value of these roles in generating the evidence base. However, with nursing care increasingly linked to patient safety and quality of care, more attention is required to address the undervaluing of nursing research. Some course for redress is with the allocation of research funding; there is inequality in research funding, with only $1.9 \%$ of higher education institution funding being awarded in the nursing and allied health professions (UK Clinical Research Collaboration, 2015), despite the composition of the NHS workforce comprising 25.6\% nurses and midwives (Nuffield Trust, 2017).

The redistribution of research funding cannot come at a whim, and must be done in parallel to increased training of nurses in the discipline of research to ensure the delivery of high quality and impactful research. Since 2005, the NIHR has funded a growing number of nurses, although allied health professions have seen the greatest increase (Medical Research Council, 2020) but the numbers are in the tens, rather than hundreds or thousands and are unlikely to make a significant dent in the proportion of clinical academics within the profession in the near future.

The importance of the GPN role in delivering primary care services has been recognised in recent policy documents (NHS England, 2017). However, the shortage of GPNs and the lack of consistent investment in their training and development has led to the introduction of new roles such as pharmacist-led services and physician associates (Nelson et al., 2019) turning attention away from investment in general practice nursing and its value. This study highlights how the impact of long-term undervaluing and investment has infiltrated the expectations of GPNs to be able to develop aspirational career pathways such as clinical academic careers.

The need to embed research skills and interest early on in nurse training is identified in this study. The introduction of a degree level qualification for nurse registration is beginning to have an impact. However, this study clearly shows how once nurses are employed in general practice the opportunities to build on this knowledge are limited and it is not portrayed as an expectation within the general practice environment, compounded by a distinct lack of role models.

This study suggests that GPNs are not aware of these opportunities and therefore are unable to apply for them. And even if they did apply, the employment model, specific to general practice, suggests that seeking funding for this sort of role would not necessarily be supported by GP partners. This is exacerbated by the current workforce crisis (NHS Digital, 2015; Bradby and McCallum, 2015) and the high proportion of current GPNs expected to retire by 2022 (33.4\%) (Bradby and McCallum, 2015).

This study is an empirical qualitative research study. Limitations include the difficulties in drawing generalisable conclusions from studies of this nature. However, they do provide a richness and depth of understanding not obtainable in quantitative studies that can be used to inform thinking. This study is also focussed on England and, therefore, might not reflect general practice nursing across the four nations of the United Kingdom, or more globally.

\section{Conclusions}

The clinical academic career in general practice nursing has the opportunity to deliver patient-derived nurse-led research that could see substantial gains in improvements to patient care including patient experience, safety and clinical outcomes. Furthermore, offering robust career pathways for the GPN workforce could also have a positive impact on the retention of experienced GPNs and attract newly qualified nurses. Traditionally, 
research has been the role of the medical profession, but as we increasingly see the nursing profession deliver complex clinical care, adopting skills and competence traditionally associated with doctors without compromising patient care, and in many cases improving it, there is little reason to think that nursing could not take greater responsibility for its own evidence base. It is important that the specific challenges for nurses working in the general practice setting are recognised and addressed to make clinical academic careers equally available to GPNs.

\section{Key points for policy, practice and/or research}

- This study provides an evidence base on the barriers and enablers to clinical academic careers in general practice nursing.

- The unique contractual arrangements for GPNs generate specific conditions for nurses working in this field that make access to clinical academic roles within primary care more difficult.

- Working with employers and instilling research confidence in nurses earlier in their education would go some way to facilitate an increase in clinical academic roles in general practice.

\section{Availability of data and materials}

The data generated by this study are available on request from the corresponding author (SJ). The data are not publicly available due to their containing information that may compromise participant anonymity.

\section{Declaration of conflicting interests}

The author(s) declared no potential conflicts of interest with respect to the research, authorship, and/or publication of this article.

\section{Ethics}

Full ethical approval for the study was granted by the Faculty of Health, Education and Life Sciences Academic Ethics Committee at Birmingham City University. All participants provided written informed consent to participate in the study. Ethics reference Jones /3061/R(A)/2019/Jan /HELS FAEC dated 15th Jan 2019.

\section{Funding}

The author(s) disclosed receipt of the following financial support for the research, authorship, and/or publication of this article: This study was funded by NHS England. The funder paid for the time of the research staff to deliver the study protocol.

\section{ORCID iDs}

Sarahjane Jones (D) https://orcid.org/0000-0003-4729-4029

Andrew Bradbury (D) https://orcid.org/0000-0002-8283-2710 


\section{References}

Aiken LH, Sloane D, Griffiths P, et al. (2017) Nursing skill mix in European hospitals: cross-sectional study of the association with mortality, patient ratings, and quality of care. BMJ Qual Safety 26(7): 559-568.

Baker C (2019) NHS Key Statistics, England, May 2019. Briefing Paper No. 7281. Available at: https:// commonslibrary.parliament.uk/research-briefings/cbp-7281/ (accessed 27 June 2021).

Baltruks D and Callaghan P (2018) Nursing, midwifery and allied health clinical academic research careers in the UK. Council of Deans of Health. Available at: https:// councilofdeans.org.uk/2018/08/new-report-nursingmidwifery-and-allied-health-clinical-academic-researchcareers-in-the-uk/ (accessed 27 June 2021).

Bhaskar RA (2008) Realist Theory of Science. London: Verso.

Bell J (2017) Life Sciences Industrial Strategy: a report to the government from the life sciences sector. Life Sciences Industrial Strategy Board. Available at: https://www.gov.uk/ government/publications/life-sciences-industrial-strategy (accessed 27 June 2021).

Bradby, M., McCallum, C. (2015) (eds) General Practice Nursing A Time Of Opportunity in the 21st Century, London: Queens Nursing Institute.

Bradby M and McCallum C (2019) General Practice Nursing in the 21st Century: A Time of Opportunity. Queen's Nursing Institute. Available at: https://www.qni.org.uk/wp-content/ uploads/2016/09/gpn_c21_report.pdf (accessed 27 June 2021).

Bradbury A, Shortland S, Jones S, Hewett F, Karen and. Clinical academic careers for general practice nurses: a rapid evidence assessment. Journal of Research in Nursing 2021; 26(5): 376-391. doi:10.1177/1744987120954261.

Braun V and Clarke V (2006) Using thematic analysis in psychology. Qual Res Psychol 3(2): 77-101.

British Medical Association (2019) Clinical academics below consultant: revision of pay arrangements in light of the NHS junior doctor contract for England - updated 2019. Available at: https://www.bma.org.uk/media/2183/bma-clinicalacademic-trainee-pay-system-england-2019.pdf (accessed 27 June 2021).

British Medical Association (2020) Medical academics' pay scales. Available at: https://www.bma.org.uk/advice/ employment/pay/medical-academics-pay (accessed 27 June 2021).

Cannaby AM, Libacao A, Hassanein E, et al. (2017) Do Chief Nurses need to be academically credible? Journal of Advanced Nursing 73(11): 2503-2505.

Centre for Reviews and Dissemination (2011) Evidence briefing on nurse endoscopy. National Institute for Health Research. Available at: https://www.york.ac.uk/media/crd/Nurse\% 20endoscopy $\% 20$ evidence $\% 20$ briefing.pdf (accessed 27 June 2021).

Danermark B, Ekstrom M, Jakobsen L, et al. (2002) Explaining Society - critical realism in the social sciences. Oxford: Routledge.

Department of Health (2012) Developing the Role of the Clinical Academic Researcher in the Nursing, Midwifery and Allied Health Professions. Crown. Available at: https://www. gov.uk/government/uploads/system/uploads/attachment_ data/file/215124/dh_133094.pdf (accessed 27 June 2021).

Dickinson J, Scott J and Edwards P (2017) UK-Wide Survey of Clinical and Health Research Fellowships. Medical Research Council. Available at: https://mrc.ukri.org/publications/ browse/clinical-and-health-research-fellowships-survey2017/ (accessed 27 June 2021).

Downing A, Morris EJ, Corrigan N, et al. (2017) High hospital research participation and improved colorectal cancer survival outcomes: a population-based study. Gut 66(1): 89-96.

Fisher D, Tang P and Knight E (2017) Survey of Medical Clinical Academic Staffing Levels 2017. Medical Schools Council. Available at: https://www.medschools.ac.uk/media/ 2026/medical-clinical-academic-staffing-levels-2017.pdf (accessed 27 June 2021).

Gibson J, Sutton M, Spooner S, et al. (2017) Ninth National GP Worklife Survey 2017. Policy Research Unit in Commissioning and the Healthcare System. Available at: https://prucomm.ac.uk/assets/uploads/Ninth-National-GPWorklife-Survey.pdf (accessed 27 June 2021).

Griffiths P, Ball J, Bloor K, et al. (2018) Nurse staffing levels, missed vital signs and mortality in hospitals: Retrospective longitudinal observational study. National Institute for Health Research. Available at: https://www.ncbi.nlm.nih.gov/ books/NBK534527/ (accessed 27 June 2021).

Health Education England (2017a) Facing the facts, shaping the future: a draft health and care workforce strategy for England to 2027. Available at: https://www.hee.nhs.uk/our-work workforce-strategy (accessed 27 June 2021).

Health Education England (2017b) The General Practice Nursing Workforce Development Plan. Available at: https:// www.hee.nhs.uk/sites/default/files/documents/The $\%$ 20 general $\% 20$ practice $\% 20$ nursing $\% 20$ workforce $\%$ 20 development \%20plan.pdf (accessed 27 June 2021).

HM Government (2019) Industrial Strategy Challenge Fund: for research and innovation. Available at: https://www.gov. uk/government/collections/industrial-strategy-challengefund-joint-research-and-innovation (accessed 27 June 2021).

Innes L (2019) General Practice Nurse education in Scotland now and in the future. Education for Primary Care 30(5): 263-266.

Larsson I, Fridlund B, Arvidsson B, et al. (2014) Randomized controlled trial of a nurse-led rheumatology clinic for monitoring biological therapy. Journal of Advanced Nursing 70(1): 164-175.

Leary A (2012) Advanced nurse practice is much more than simply role substitution. Nursing Standard 26(51): 32.

Leary A, Cook R, Jones S, et al. (2016) Mining routinely collected acute data to reveal non-linear relationships between nurse staffing levels and outcomes. BMJ Open 6(12): e011177.

Leary A, Maclaine K, Trevatt P, et al. (2017) Variation in job titles within the nursing workforce. Journal of Clinical Nursing (23-24): 4945-4950.

MacWilliams BR, Schmidt B and Bleich MR (2013) Men in nursing. American Journal of Nursing 113(1): 38-44.

Medical Research Council (2020) Career Development Award $(C D A)$ : Transition to independence. Available at: https:// mrc.ukri.org/skills-careers/fellowships/non-clinicalfellowships/career-development-award-cda-transition-toindependence/ (accessed 27 June 2021).

NHS Digital (2018) General and Personal Medical Services, England. Available at: https://files.digital.nhs.uk/17/ 93CB4C/Sept \%202018\%20Report\%20v3.pdf (accessed 27 June 2021).

NHS England (2016) General Practice: Forward View. Available at: https://www.england.nhs.uk/wp-content/ uploads/2016/04/gpfv.pdf (accessed 27 June 2021). 
NHS England (2017) General Practice: Developing confidence, capability and capacity. NHS England Publications Gateway Reference number: 06870. Available at: https://www. england.nhs.uk/wp-content/uploads/2018/01/generalpractice-nursing-ten-point-plan-v17.pdf (accessed 27 June 2021).

Nelson PA, Bradley F, Martindale AM, et al. (2019) Skill-mix change in general practice: a qualitative comparison of three 'new' non-medical roles in English primary care. British Journal of General Practice 69(684): e489-e498.

Nijjar SK, D'Amico MI, Wimalaweera NA, et al. (2017) Participation in clinical trials improves outcomes in women's health: a systematic review and meta-analysis. British Journal of Obstetrics and Gynaecology 124(6): 863-871.

Nuffield Trust (2017) The composition of the NHS workforce. Available at: https://www.nuffieldtrust.org.uk/chart/thecomposition-of-the-nhs-workforce (accessed 27 June 2021).

O'Brien BC, Harris IB, Beckman TJ, et al. (2014) Standards for Reporting Qualitative Research: A Synthesis of Recommendations. Academic Medicine 89(9): 1245-1251.

Pirret AM, Neville SJ and La Grow SJ (2015) Nurse practitioners versus doctors diagnostic reasoning in a complex case presentation to an acute tertiary hospital: a comparative study. International Journal of Nursing Studies 52(3): 716-726.

Rolewicz L and Palmer B (2019) The NHS workforce in numbers: Facts on staffing and staff shortages in England. Nuffield Trust. Available at: https://www.nuffieldtrust.org. uk/resource/the-nhs-workforce-in-numbers\#2-what-is-theoverall-shortfall-in-staff-in-the-nhs (accessed 27 June 2021).

UK Clinical Research Collaboration (2006) Developing the best research professionals. Qualified graduate nurses: recommendations for a clear career pathway for those nurses involved in clinical research preparing and supporting clinical academic nurses of the future. Available at: http://www.ukcrc. org/wp-content/uploads/2014/07/Nurses-report-August-07Web.pdf (accessed 27 June 2021).

UK Clinical Research Collaboration (2015) UK Health Research Analysis 2014. Medical Research Council. Available at: http://www.ukcrc.org/wp-content/uploads/ 2015/08/UKCRCHealthResearchAnalysis2014-WEB.pdf (accessed 27 June 2021).

Watson R, McDonagh R and Thompson DR (2017) h-indices: an update on the performance of professors in nursing in the UK. Journal of Advanced Nursing 72(5): 999-1001.

Watson R and Thompson DR (2008) Professors as leaders. Journal of Clinical Nursing 17(8): 981-982.

Sarahjane Jones is an associate professor in patient safety at Staffordshire University. Her research interests are in improving the safety of healthcare and workforce welfare including clinical academic careers for non-medical professionals.

Andrew Bradbury is a health tutor at CU Scarborough and is currently studying for a $\mathrm{PhD}$ at Nottingham Trent University.

Sue Shortland is an associate professor at Birmingham City University and leads the fundamentals of general practice nursing programme. Before entering academia, Sue was an advanced nurse practitioner in general practice.

Fraser Hewett is a full-time NHS GP partner with clinical interests in mental health and minor surgery, who is keen on primary care research, and local health service development.

Karen Storey is Primary Care Nursing Lead at NHS England and Improvement, leading on the General Practice Nursing Ten Point Plan which aims to recruit, retain and return nurses to general practice. 